\title{
Le lecteur fantôme
}

In Koli Jean Bofane

URL : https://journals.openedition.org/coma/5981

DOI : 10.4000/coma.5981

ISSN : 2275-1742

\section{Éditeur}

Institut des textes \& manuscrits modernes (ITEM)

\section{Référence électronique}

In Koli Jean Bofane, «Le lecteur fantôme », Continents manuscrits [En ligne], 15 | 2020, mis en ligne le 15 octobre 2020, consulté le 13 janvier 2023. URL : http://journals.openedition.org/coma/5981 ; DOI https://doi.org/10.4000/coma.5981

Ce document a été généré automatiquement le 13 janvier 2023.

\section{(c) (i) (2)}

Creative Commons - Attribution - Pas d'Utilisation Commerciale - Pas de Modification 4.0 International - CC BY-NC-ND 4.0

https://creativecommons.org/licenses/by-nc-nd/4.0/ 


\title{
Le lecteur fantôme
}

\author{
In Koli Jean Bofane
}

\section{NOTE DE L'ÉDITEUR}

Né en 1954 à Mbandaka, « à l'intersection du fleuve Congo et du méridien de l'équateur », In Koli Jean Bofane vit en Belgique. Il publie en 1996 le recueil de récits pour enfants Pourquoi le lion n'est plus le roi des animaux (Gallimard), puis en 2008 le roman Mathématiques congolaises, qui a reçu le prix Jean-Muno, le prix de la Scam et le grand prix littéraire d'Afrique noire de l'Adelf. Congo Inc. Le Testament de Bismarck, son deuxième roman, reçoit en 2015 le prix des Cinq Continents de la Francophonie. Son troisième roman, La Belle de Casa, a été publié en 2018 chez Actes Sud. Ses ouvrages ont été traduits aux États-Unis, en Italie, en Allemagne, au Brésil, en Corée et en Slovénie. Il nous livre ici un texte sur son rapport aux livres et à la bibliothèque, teinté de sacré, d'humour, d'effroi et de fantastique. 


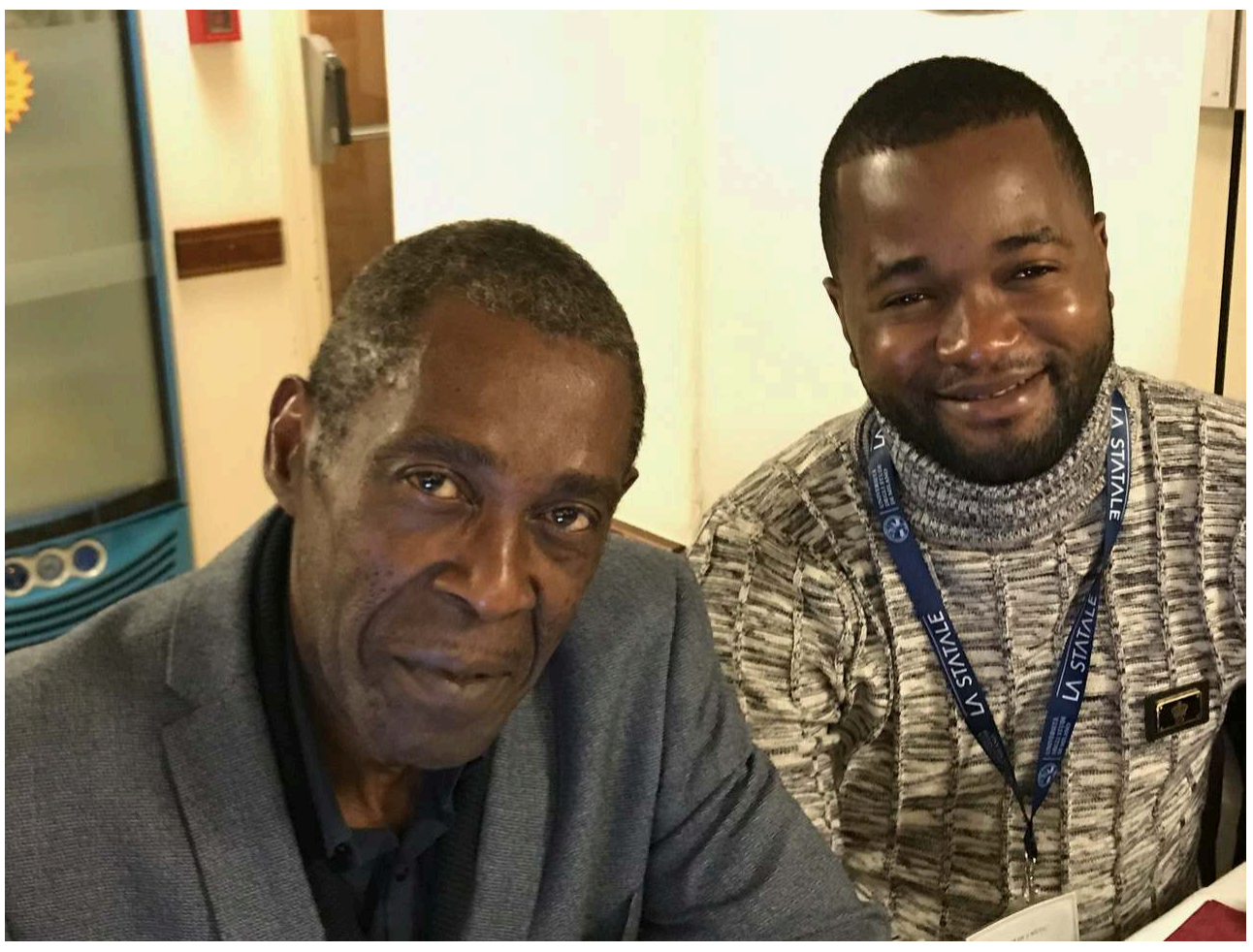

In Koli Jean Bofane (à gauche) et Richard Ali Mutu (au deuxième plan)

photographie de Silvia Riva

Sans doute que si je n'étais pas né à l'intersection du fleuve Congo et du méridien de l'équateur, jamais la lecture n'aurait pu revêtir cet aspect sacré tel que je l'ai toujours perçu. Parce qu'il faut savoir, dans ce pays, et surtout dans les bourgades de l'Équateur où j'ai vécu, juste après l'Indépendance, que le livre était rare, l'écrit ne courait pas les rues. Avant l'Indépendance, celui qui savait lire, portait, d'ailleurs, une chemise blanche, la cravate, souvent un chapeau et le Blanc lui donnait le grade d' "évolué » ; un statut à part entière, que l'on ne peut obtenir que capable de déchiffrer un alphabet de base. Alors, comment s'étonner que de temps en temps, le vieil oncle, à l'heure où le soleil rougit le ciel, après un dur labeur, devant une bière, qu'il puisse chausser sa paire de lunettes et fasse comme celui qui lit couramment, même si le journal qu'il a récolté à son travail - il y a bien trois mois de cela - est fixé pendant de très longues minutes. Jamais, autour de lui, personne ne commettra l'hérésie de se poser la question si l'oncle sait effectivement lire ou ne fait que décoder chaque caractère sur le papier et tenter de comprendre un mot. L'important n'est pas là, ce qui compte, c'est de poser un geste solennel aux yeux de tous, accomplir la performance qui consiste à incarner celui qui détient une sagesse mystérieuse, telle une idole Tshokwe ou Kuba sculptée dans le bois, adoptant la posture immobile mais capable de prodiges, pouvant même se muer en démiurge, en cas de besoin. Le Congolais connaît le pouvoir de l'écriture et de la lecture. Ne dit-on pas au pays de Mobutu que le Blanc vole avec le stylo? Une croix apposée sur un document illisible et le lendemain un quidam se présente vous signifiant que la terre sur laquelle vous posez vos pieds, le pays autour et votre personne sont désormais à sa disposition. Forcément dans un environnement ayant bénéficié d'une telle expérience, le livre ne sera pas seulement un livre, il seras un grimoire ou quelque chose d'approchant. Il n'est pas rare de croiser dans les avenues 
poussiéreuse de Kinshasa des êtres traçant la ligne $11^{1}$, un livre unique sous le bras, un ouvrage censé contenir la clé de l'existence. Dans un tel contexte, enfant, j'avais hâte d'aussi poser ce geste ; le regard vif plongé sur deux pages ouvertes et balayant les mots l'un après l'autre, un air concentré comme celui arboré par ces individus de renom que l'on ne rencontre que dans les dictionnaires.

2 Lorsqu'on mesure un peu plus d'un mètre, une bibliothèque avec ses étagères pleines de livres est comme la paroi d'une montagne où la connaissance se déclinerait dans les couleurs des tranches des livres portant des labels tels Gallimard, Mercure de France ou Fleuve Noir. Je passais devant celles de papa tous les jours. Mes yeux lorgnaient vers des noms de dames et messieurs comme Flaubert, Stendhal, Sand, Faulkner; des titres aussi, tels, L'Arrache-cœur, Bonjour tristesse, Quand sonne le glas, J'irai cracher sur vos tombes, Les Raisins de la colère. Il était clair pour moi que, savoir qui était cet individu qui arrachait les cœurs, était devenu une obsession, et comprendre pourquoi quelqu'un cracherait sur une tombe, déjà, j'en était conscient, c'était découvrir une grande partie de la complexité de ce monde. Je rongeais mon frein mais jamais je n'aurais eu l'idée de m'emparer d'un livre sans la permission de celui qui régnait sur cette montagne du savoir, mon père en l'occurrence.

3 Le sacré ne se transmet pas ainsi, de façon désinvolte. Il faut un officiant, un rite approprié, une ou des paroles doivent être prononcées. Puis, une guerre éclata, la rébellion muleliste. Elle faisait rage en cette année 1964. On envoya mon frère et ma sœur en Belgique et je restai seul en compagnie des parents. Si, malgré une forte tentation, je ne me suis jamais laissé aller à m'emparer d'un bouquin afin de tenter de le lire en cachette, c'est que j'aspirais à devenir un Grand-Prêtre de la lecture et pas moins. Dans ce cas, cela nécessite une initiation comme pour ces jeunes gens, obligé d'intégrer le séminaire pour pouvoir, un jour ou l'autre, accéder à la papauté ou comme ces enfants du Tibet au crâne rasé, portant robes couleur oranger ou safran, que j'avais vu dans le National Geographic Magazine. Avec la guerre, papa fut mis dos au mur. Il fallait absolument transcender la morbidité ambiante et atteindre des niveaux supérieur de pensée. Il m'emmena devant la paroi faite de livres et qui ressemblait à la montagne où vivaient Hassan ibn al-Sabbah, le vieil homme de la montagne, et ses hachischins dont m'avait parlé papa, sur la véranda, un soir, au son des grillons, des hululements de rapaces nocturnes et des sifflements de chauve-souris. Il me fit me planter devant l'autel. De lui-même, mon visage se leva vers le haut comme lors d'une assomption. Papa tendit, alors, le bras, prononça une parole pleine de sens: "Choisis ! ». C'était comme quand le Christ avait dit à ce type : "Lazare lève-toi ! "

Il faut dire que mon choix fut net. Lorsque le destin vous ouvre de façon grandiose les portes de l'avenir, il faut être déterminé. Les divinités sont susceptibles et n'aiment pas celui qui tergiverse et qui doute de sa foi, autrement dit, de leurs capacités. Il faut se rappeler aussi que je suis né en pleine colonisation. Le Blanc squattait tout en ce tempslà, surtout les rayons de la bibliothèque familiale. Parmi ces écrivains et penseurs, l'un d'eux avait tout naturellement attiré mon intérêt. Il s'agissait de Zola Émile. Le terme zola en lingala signifiant «amour » et le titre du roman étant "Nana " - un prénom purement congolais - je fus persuadé qu'un compatriote avait méprisé les concepts d'esclavagisme et de colonialisme pour se hisser, par je ne savais quel prodige, au-delà de la troisième étagère de la bibliothèque; comme certains de ceux qui venaient de m'accorder une indépendance toute fraîche. Après une page et demi je dus bien me résoudre à accepter l'évidence, encore une fois, un diable de Français affublé d'un faux 
nom, optant pour un titre racoleur, m'avait complètement roulé dans la farine : Nana n'avait rien avoir avec le Congo. L'arnaque fut comme un défi et j'engloutis l'ouvrage sans même reprendre mon souffle, me délectant des courbes pulpeuses de Nana, me substituant à un de ses amants lorsqu'il lui arrivait de lui baiser la main, frissonnant lorsqu'une mèche de ses cheveux de rousse voletait à la caresse d'une bise légère, rue Chaussée d'Antin.

5 Lorsque vous devenez Grand-Prêtre de la lecture, votre vie ne vous appartient plus. C'est aux écrits à qui vous vous vouerez. Enfant, je lisais tout ce qui me tombait sous la main; non seulement, les livres et toutes les quatrièmes de couverture, mais les magazines également, les bandes dessinées, les pages du dictionnaire, les notices des médicaments et leurs contre-indications, celles sur les boîtes de sardines et de flocons d'avoines au petit déjeuner. L'envoûtement me fera lire jusqu'aux pages blanches et jaunes du bottin téléphonique en passant par les étiquettes des vêtements ou la marque et les caractéristiques du pneu d'une voiture pendant que la roue tourne sur elle-même sur l'asphalte. Cela me faisait bien un peu peur, tout cela, j'avais pressenti cette addiction formidable mais le chemin qu'emprunte le Grand-prêtre ne permet pas la volte-face, il faut savoir, c'est contraignant, un sacerdoce.

Les années passèrent, je devins adulte et mon rythme de lecture se maintint jusqu'au jour ou une autre guerre éclata. Au Rwanda, cette fois-ci. Et elle me traumatisa à tel point que je passai de l'autre côté du miroir et m'attelai, moi aussi, à gravir les degrés de la montagne de la connaissance qu'étaient les étagères de la bibliothèque de mon père. Je vous ai révélé la vertu que possèdent les livres face à la guerre. Il me fallait en écrire un comme ceux que je lisais durant les temps d'une des miennes, la rébellion de Pierre Mulele. Du coup, en passant à l'écriture, je découvrais le lecteur. Dès mon premier roman, Mohammed Moulessehoul, alias, Yasmina Khadra, croisé à mon premier salon, m'entretint sur l'importance primordial de se constituer un lectorat. Sans lui, tu es mort, crus-je entendre. En tant que Grand-Prêtre de la lecture je ne peux ignorer les symptômes de l'addiction à la lecture frappant le lecteur; les émotions violentes, les vertiges que cela provoque, les questionnements que cette activité suscite. Ne parlons pas des effets secondaires en cas de fiction trop prenante: une distraction perpétuelle, les fous rires qu'il faut étouffer dans le métro lorsque l'auteur a réussi par le truchement d'un personnage à ce que vous puisiez rire de vous-même, le regards de l'autre. C'est sur ces manifestations que je comptais réaliser la quête que me conseillait mon frère écrivain. Sans le lecteur, écrire ne sert à rien. Conscient de cette donne, j'avais pris les devants avant même de publier quoi que ce soit mais je devais savoir si le lecteur allait volontiers m'agréer dans sa psyché.

7 Les premiers parmi eux, je les désignais moi-même. Comme mon père l'avait fait avant moi, il me fallait user d'un mot sacré aussi puissant que « Choisis! ». « Lis ! » leur disaisje. Ils obtempéraient immédiatement et lisaient car je dois vous dire qu’à cette époque, juste débarqué en Belgique sans papiers et aucune légitimité, avec beaucoup d'audace, je pratiquai le travail clandestin en tant que videur de boîte. Mes premiers lecteurs, je les choisissais parmi les clients quittant la discothèque aux aurores lorsque l'alcool, le désir pour le ou la partenaire et les sens étaient en pleine sollicitation et que l'esprit est à la limite de conserver sa cohérence. Si le client brusquement absorbé, lit le texte jusqu'au bout sans tenir compte des jérémiades de la copine qui veut rentrer, c'était gagné. Je choisissais pour faire ressortir le caractère divin de la démarche, celui que je soupçonnais n'avoir jamais été frappé par la littérature, afin que mon texte puisse le 
remplir et agir telle une révélation. Des lecteurs, mes romans réussissent à les débusquer. Ils achètent mes livres, suivent de près mes interviews, m'accompagnent partout où je me trouve à travers le monde, sollicitent des dédicaces. Je suis à peu près rassuré, sachant que je peux faire confiance à l'assuétude que génère la littérature.

Très vite, j'ai découvert le lecteur fantôme. Il est apparu de façon inattendue, dès la rédaction des premières lignes de mon premier roman. Je m'attendais à tout sauf à la présence d'une ombre occupant la chaise à ma droite. Un ami m'avait prêté son château situé dans la forêt du Luxembourg, en Belgique. Les planchers et les marqueteries craquaient sans arrêt et les portraits des ancêtres me fixaient dans la pénombre. J'y était totalement seul avais-je pensé, et c'est à ce moment-là, qu'il est apparu. Immobile la plupart du temps, il ne me parlait jamais, m'obligeant juste à essayer de deviner sa pensée. À son air, un peu emprunté, je sentais qu'il ne recherchait pas ma sympathie mais je le pressentais indispensable, quelque part, dans l'édification de mon œuvre future. C'est avec lui que je discutais des problématiques que j'abordais. Lui et moi, nous nous livrions à des joutes cérébrales des nuits entières. Parfois pour tenter de le désorienter, je n'écrivais pas tout, le laissant deviner le fond de ma pensée. Il était brillant, restait muet mais ses réflexions à lui apparaissaient toujours lors des rencontres dans la bouche d'un lecteur ou d'un critique littéraire plus performant que les autres.

Le lecteur fantôme est une entité cynique aussi. Un mot pouvait me fuir pendant des mois et durant ce laps de temps, je sentais qu'il se moquait de moi et pour accentuer ma frustration face à ma propre carence, finalement, il me soufflait le mot en ricanant. C'était d'ailleurs les seuls sons qu'il émettait : des ricanements. Son attachement pour moi aurait pu me faire croire à un amour inconditionnel pour ma personne, comme les lecteurs en chair et en os déversent sur moi, mais lui, ses motivations envers moi étaient peu claires. Ne sachant pas ce qu'il me voulait, je ne me dévoilais jamais complètement et ne pouvais surtout pas m'octroyer le luxe de l'angoisse de la page blanche. Il ne fallait surtout pas faire transparaître mes faiblesses. Je me méfiais de lui et pour tout avouer, je ne parviens toujours pas à l'aimer comme je devrais peut-être le faire. De toute façon, je ne pourrai jamais m'en débarrasser. Une amie devineresse est passée me voir l'autre jour, m'a dit qu'elle voyait bien « quelque chose » de flou auprès de moi mais elle me dit aussi qu'il n'y avait aucune raison que je m'inquiète de sa présence, le type avait l'air de me ressembler comme deux gouttes d'eau, prétendaitelle, mais j'en doutais fortement. La littérature m'avait produit, moi, et inconsciemment, je savais qu'elle ne pouvait produire ce genre de fantôme. Même Shakespeare, imaginant le spectre du père de Hamlet, aurait hésité à en créer un qui ressemble à mon lecteur-fantôme.

\section{NOTES}

1. Tracer la ligne 11 : expression populaire fondée sur le rapprochement entre la silhouette des jambes (deux barres parallèles) et la graphie du chiffre 11. L'expression signifie accomplir une longue distance à pied par manque d'argent pour payer le ticket de transport. 
INDEX

Mots-clés : prose, inédit, littérature congolaise, livre, bibliothèque, lecture, lecteur

\section{AUTEUR}

\section{IN KOLI JEAN BOFANE}

Auteur congolais, né en RDC en 1954 et vivant en Belgique. 